\title{
Development of gluten-free breads started with chia and flaxseed sourdoughs fermented by selected lactic acid bacteria
}

\author{
Stefanía Dentice Maidana ${ }^{\mathrm{a}, \mathrm{b}}$, Susan Finch $^{\mathrm{b}}$, Marisa Garro ${ }^{\mathrm{a}}$, Graciela Savoy ${ }^{\mathrm{a}}$, Michael Gänzle ${ }^{\mathrm{b}, \mathrm{c}}$, \\ Graciela Vignolo ${ }^{\mathrm{a}, *}$
}

${ }^{a}$ Centro de Referencia para Lactobacilos (CERELA), Chacabuco 145, 4000, Tucumán, Argentina

${ }^{\mathrm{b}}$ Department of Agricultural, Food and Nutritional Science, University of Alberta, 4-10 Ag/For, Edmonton, Alberta, T6G 2P5, Canada

${ }^{\mathrm{c}}$ Hubei University of Technology, College of Bioengineering and Food Science, Wuhan, Hubei, PR China

\section{A R T I C L E I N F O}

\section{Keywords:}

Gluten-free

Chia

Lactic acid bacteria

\begin{abstract}
A B S T R A C T
Gluten-free baked goods are a challenge for biotechnologists and nutritionists because their low baking characteristics. Lactic acid bacteria (LAB) were identified and characterized from chia flour and spontaneously fermented sourdough. Based on techno-functional and safety properties, Weissella cibaria CH28 together with Lactobacillus plantarum FUA3171 and Lactobacillus fermentum FUA3165 from fermented sorghum, were used as inoculants to ferment chia and flaxseed sourdoughs used for sorghum gluten-free breadmaking. Compared to chemically acidified control, fermentation products present in oilseed sourdoughs after $24 \mathrm{~h}$ showed a marked increase of lactate, xylose, arabinose, free amino acids and hydrogen peroxide while glucose was undetectable. Sorghum-based laboratory breads manufactured with different percentages $(0-40 \% \mathrm{w} / \mathrm{w})$ of oilseeds sourdoughs fermented by $W$. cibaria $\mathrm{CH} 28$ and lactobacilli showed that those started with 30 and $40 \%$ of sourdoughs significantly improved specific volume and visual appearance compared to $100 \%$ sorghum breads. Sensory evaluation of gluten-free sorghum breads showed $40 \%$ of replacement as the most accepted by panelists who were also able to discriminate breads inoculated with fermented and unfermented chia dough. The use of chia sourdoughs fermented with selected autochthonous LAB strains as ingredient for gluten-free breadmaking, will have an impact in baked foods functionality and healthiness.
\end{abstract}

\section{Introduction}

Chia (Salvia hispanica L.) is an annually cultivated plant that belongs to the mint family (Labiatae). Chia seeds were an important staple food for pre-Columbian societies in Central America (Ayerza \& Coates, 2005). Flax (Linum usitatissimum L.) is produced as oilseed and fiber crop; its cultivation originated in the eastern Mediterranean region and goes back as far as 5000 B.C (Bernacchia, Preti, \& Vinci, 2014). Chia seeds and flaxseeds constitute a good source of proteins (24 and $30 \%$, respectively), dietary fiber and polyunsaturated fatty acids (25\% and $23 \%$, respectively) of which around 60-63\% (chia) and 70\% (flaxseed) represent $\omega-3$ fatty acid $\alpha$-linolenic acid (Hussain, Anjum, Alamri, Mohamed, \& Nadeem, 2013; Muñoz, Cobos, Diaz, \& Aguilera, 2013). Both oilseeds are rich in natural antioxidant compounds, vitamins (Bvitamins, tocopherols and niacin), and minerals (Bernacchia et al., 2014; Marineli et al., 2014; Muñoz et al., 2013; Wang et al., 2017). Compared to wheat (72\%), chia and flaxseed exhibit a lower carbohydrates content ( $42 \%$ and $29 \%$, respectively). Bioactive components of flaxseed and chia seeds benefit human health; consumption was reported to improve biological markers related to dyslipidemia, inflammation, cardiovascular disease, glucose homeostasis, and diabetes without adverse effects (Goyal, Sharma, Upadhyay, Gill, \& Sihag, 2014; Muñoz et al., 2013; Parker, Schellenberger, Roe, Oketch-Rabah, \& Calderón, 2018). Despite their nutritional benefits, they are rarely used in food production. The use of chia and flaxseed as complement to cereal flours for production of baked goods can improve organoleptic and nutritional properties based on their polysaccharides and fatty acids content (Goyal et al., 2014; Hussain et al., 2013; Iglesias-Puig \& Haros, 2013; Lamo \& Gómez, 2018; Sandri, Santos, Fratelli, \& Capriles, 2017). Oilseeds are particularly promising in gluten-free (GF) baking, where hydrocolloids and proteins can serve as functional replacement for gluten (Zannini, Jones, Renzetti, \& Arendt, 2012). Although the low baking characteristics of GF flours, fermentation improves and diversifies the quality of GF breads (Moroni, Dal Bello, \& Arendt, 2009).

\footnotetext{
* Corresponding author. Centro de Referencia para Lactobacilos (CERELA), Chacabuco 145, 4000, Tucumán, Argentina.

E-mail addresses: sdentice@cerela.org.ar (S. Dentice Maidana), sfinch@ualberta.ca (S. Finch), mgarro@cerela.org.ar (M. Garro), gsavoy@cerela.org.ar(G. Savoy), mgaenzle@ualberta.ca (M. Gänzle),vignolo@cerela.org.ar (G. Vignolo).
} 
Sourdough fermentation has the potential to exploit the technological, nutritional, functional and sensory features of wheat and nonwheat flours (Rizzello, Calasso, Campanella, De Angelis, \& Gobbetti, 2016). In particular, dough workability, bread structure, organoleptic and nutritional properties of raw flours are improved. Furthermore, sourdough fermentation increases the content of bioactive compounds and the uptake of minerals, decreasing the level of anti-nutritional factors and the value of the glycemic response (Gobbetti, Rizzello, Di Cagno, \& De Angelis, 2014). Thus, the use of the sourdough in GF baking may be the new frontier for improving the quality, safety and acceptability of GF baked products (Coda, Cagno, Gobbetti, \& Rizzello, 2014). The stable sourdough microbiota consists of particular lactic acid bacteria (LAB) species, mostly facultative and obligate heterofermentative strains, due to their highly adapted carbohydrate metabolism and dedicated amino acid assimilation in the sourdough environment, their impact on the biochemistry of sourdough and product quality being increasingly understood (De Vuyst et al., 2014; Gänzle, 2014; Gänzle \& Zheng, 2018). Products prepared with legumes and oilseeds are generally not fermented, however LAB use in sourdough fermentation requires the selection of competitive starter cultures that beneficially affect product quality (Coda et al., 2014; Rizzello, Lorusso, Montemurro, \& Gobbetti, 2016; Sáez, Hébert, Saavedra, \& Zárate, 2017). Moreover, the beneficial effect of sourdough fermentation on product quality not only depends on the metabolic traits of LAB but also on the enzymatic activity of the substrate (Gänzle, 2014); endogenous enzyme activities of chia and flaxseed are poorly characterized. Therefore, to exploit the potential of these flour matrices, the selection of adequate starter cultures is needed (Coda et al., 2014; Denkova, Ilieva, Denkova, Georgieva, \& Krastanov, 2014a). Accordingly, LAB were recently isolated, identified and characterized from spontaneous chia sourdough fermentation (Dentice Maidana et al., 2020). The bacterial profile revealed Weissella (W.) cibaria, Lactobacillus (L.) rhamnosus and Lactococcus ( $L c$.) lactis as the dominant LAB at the final propagation stages of chia sourdough. The aim of the present study was the use of chia and flaxseed sourdough, as inoculants of laboratory breads formulated with gluten-free sorghum flour.

\section{Materials and methods}

\subsection{Bacterial strains and growth conditions}

W. cibaria $\mathrm{CH} 28$ and Lc. lactis $\mathrm{CH} 179$ were previously isolated from chia sourdoughs and selected for their techno-functional and safety properties (Dentice Maidana et al., 2020). In addition, L. plantarum FUA3171 and L. fermentum FUA3165 isolated from sorghum-based sourdoughs (Sekwati-Monang \& Gänzle, 2011) from the strain collection of the Food Microbiology Laboratory, University of Alberta (Canada) were used for comparative purposes. Strains were cultivated overnight in MRS- 5 medium at $30{ }^{\circ} \mathrm{C}$ (Meroth, Walter, Hertel, Brandt, \& Hammes, 2003). Enumeration of LAB was carried out by plating onto MRS (Oxoid) at $30{ }^{\circ} \mathrm{C}$ for $48 \mathrm{~h}$.

\subsection{Laboratory sourdough preparation using chia and flaxseed flours}

Chia (Sturla ${ }^{\mathrm{TM},}$ Argentina) and flaxseed (from local market) flours used in this study were fermented according to the formula: $50 \mathrm{~g}$ of flour $+100 \mathrm{~mL}$ of tap water $+50 \mathrm{~mL}$ of each selected suspension of $\mathrm{LAB}$ strain or combinations. For inoculation, selected LAB strains were grown overnight in MRS-5 medium, centrifuged (15000 g; $15 \mathrm{~min}$ ), washed with sterile distilled water and resuspended in $50 \mathrm{~mL}$ of sterile water to reach an initial cell density of $7 \log \mathrm{CFU} / \mathrm{g}$ in the doughs. Inoculated doughs were fermented for $24 \mathrm{~h}$ at $30{ }^{\circ} \mathrm{C}$. For each flour, uninoculated controls doughs were chemically acidified to $\mathrm{pH} 4.0$ with 4-parts lactic acid (80\%) and 1-part glacial acetic acid (100\%). Before and after fermentation, samples $\mathrm{pH}$ and LAB counts were determined. For each condition, two independent fermentation were carried out.

\subsubsection{Analysis of water-soluble carbohydrates and organic acids}

For quantification of water-soluble carbohydrates, $2 \mathrm{~g}$ of dough were mixed with $6 \mathrm{~mL}$ of distilled $\mathrm{H}_{2} \mathrm{O}$ and incubated for $2 \mathrm{~h}$ at $80^{\circ} \mathrm{C}$. Dough particles were removed by centrifugation (5000 $\mathrm{g} ; 10 \mathrm{~min}$ ). Polysaccharides in the supernatant were precipitated with ethanol (70\%) during $3 \mathrm{~h}$ at $4{ }^{\circ} \mathrm{C}$ and collected by centrifugation. The pellet was resuspended in distilled water $(1 \mathrm{~mL})$ and hydrolyzed with the same volume of $2 \mathrm{M} \mathrm{H}_{2} \mathrm{SO}_{4}$ by incubation at $80^{\circ} \mathrm{C}$ for $2 \mathrm{~h}$; after centrifugation (5000 $\mathrm{g} ; 10 \mathrm{~min}$ ) monosaccharides were recovered form supernatant for HPLC determination. Organic acids were analyzed after addition of $7 \%$ perchloric acid to dough supernatant $(1: 1 \mathrm{v} / \mathrm{v})$, overnight incubation at $4{ }^{\circ} \mathrm{C}$ to precipitate proteins, and centrifugation (10000 $\mathrm{g} ; 10 \mathrm{~min}$ ). Organic acids and monosaccharides were determined by HPLC equipped with an Agilent 1200 series LC system (Agilent Technologies, Palo Alto, CA, USA) using an Aminex 87H column (300 mm $\times 7.8 \mathrm{~mm}$, Bio-Rad, Mississauga, Canada) coupled with a refractive index detector. Elution was carried out at $70{ }^{\circ} \mathrm{C}$ with a flow rate of $4 \mathrm{~mL} / \mathrm{min}$ using $5 \mathrm{mM} \mathrm{H}_{2} \mathrm{SO}_{4}$ as a mobile phase. Xylose, arabinose, glucose, fructose, galactose, fucose, rhamnose, lactate, acetate and ethanol were used as external standards. Fermentation quotient (FQ) was determined as the molar ratio between lactic and acetic acids.

\subsubsection{Determination of free amino acids}

Free amino acids (FAA) were determined in fermented doughs by the ninhydrin test as described by Thiele, Gänzle, \& Vogel. (2002). Samples (250 mg of fermented dough) were mixed with $250 \mu \mathrm{L}$ of $7 \%$ perchloric acid. After overnight precipitation at $4{ }^{\circ} \mathrm{C}$, samples were centrifuged (15000 $\mathrm{g} ; 10 \mathrm{~min})$ and the supernatant $(100 \mu \mathrm{L})$ was mixed with $20 \mu \mathrm{L} 3 \mathrm{M} \mathrm{KCl}$ to precipitate perchloric acid. After $1 \mathrm{~h}$ at room temperature, the precipitate was removed by centrifugation $(15000 \mathrm{~g}$; $10 \mathrm{~min})$. The supernatant $(10 \mu \mathrm{L})$ was diluted $(1: 10 \mathrm{v} / \mathrm{v})$ with reagent 1 (dehydrated $\mathrm{Na}_{2} \mathrm{HPO}_{4}, 5.0 \mathrm{~g} ; \mathrm{KH}_{2} \mathrm{PO}_{4}, 6.0 \mathrm{~g}$; ninhydrin, $0.5 \mathrm{~g}$; fructose, $0.3 \mathrm{~g}$ in $100 \mathrm{~mL}$ of bidistilled $\mathrm{H}_{2} \mathrm{O}, \mathrm{pH} 6.7$ ) and mixed with $190 \mathrm{~mL}$ of bidistilled water and heated $\left(100{ }^{\circ} \mathrm{C}, 16 \mathrm{~min}\right)$. Then, $500 \mu \mathrm{L}$ of reagent 2 $\left(\mathrm{KIO}_{3}, 0.2 \mathrm{~g}\right.$ dissolved in $60 \mathrm{~mL}$ of bidistilled water and $40 \mathrm{~mL}$ of $96 \%$ ethanol) were added. The sample was thoroughly mixed and absorbance (A) at $570 \mathrm{~nm}$ was measured in a microtiter plate reader. A calibration curve was prepared using glycine as standard. FAA concentration (mg/100 g dough) was calculated by $A_{570}$ of test solution/ mean $A_{570}$ of standard) $\times 2$ x dilution. The test was performed in triplicate.

\subsubsection{Quantification of peroxides}

Total peroxides were quantified by the method of Sinha (1972) modified by Mohammad (2015). Fresh dough samples (1 g) were vortexed with $2 \mathrm{~mL}$ deionized water for $20 \mathrm{~s}$. After centrifugation $(5000 \mathrm{~g}$; $5 \mathrm{~min}$ at $4{ }^{\circ} \mathrm{C}$ ), the supernatant was mixed with equal volume of potassium dichromate (5\%) in acetic acid solution $(1: 3, \mathrm{v} / \mathrm{v})$ and heated at $80{ }^{\circ} \mathrm{C}$ for $10 \mathrm{~min}$. After cooling, samples absorbance was measured at $570 \mathrm{~nm}$ (Varioskan, Thermofisher, Ottawa, Canada). $\mathrm{H}_{2} \mathrm{O}_{2}$ was used to establish a standard curve. The test was performed in triplicate.

\subsection{Gluten-free bread making}

Sorghum flour was used as a base for gluten-free breads preparation. A reference formulation consisting of 100 parts sorghum flour, 90 parts of water, 2 parts of xanthan gum, 2 parts of instant dry yeast, 1 part of salt and 0.5 part of sucrose was used to prepare breads. All ingredients were purchased from a local supermarket. As shown in Table 1, in addition to the reference bread with $100 \%$ sorghum, reads were prepared using different percentages $(5,10,15,20,30$, and $40 \%)$ of chia and flaxseed 24 h-sourdough fermented by $W$. cibaria CH28 and $L$. plantarum FUA3165 + L. fermentum FUA3171. Non-fermented flours (chia and flaxseed flours + water in same percentages) were also included. For bread preparation, commercial instant dry yeast was first activated for $10 \mathrm{~min}$ by dissolving in tap water + sucrose and allowed 
Table 1

Formula for preparation of sorghum-based breads inoculated with $W c$ and $L p$ $+L f$ sourdoughs.

\begin{tabular}{llllllll}
\hline \multicolumn{2}{c}{ Sourdough } & $\begin{array}{c}\text { Sorghum } \\
\text { flour }(\mathrm{g})\end{array}$ & Water $(\mathrm{g})$ & $\begin{array}{l}\text { Xanthan } \\
\text { Gum }(\mathrm{g})\end{array}$ & Yeast $(\mathrm{g})$ & Salt $(\mathrm{g})$ & Sugar $(\mathrm{g})$ \\
\hline$(\%)$ & $(\mathrm{g})$ & & & & & & \\
\hline 0 & 0 & 200 & 180 & 4 & 4 & 2 & 1 \\
5 & 10 & 190 & 180 & 4 & 4 & 2 & 1 \\
10 & 20 & 180 & 180 & 4 & 4 & 2 & 1 \\
15 & 30 & 170 & 180 & 4 & 4 & 2 & 1 \\
20 & 40 & 160 & 180 & 4 & 4 & 2 & 1 \\
30 & 60 & 140 & 135 & 4 & 4 & 2 & 1 \\
40 & 80 & 120 & 135 & 4 & 4 & 2 & 1
\end{tabular}

to rehydrate at room temperature. Then, different percentages of chia and flaxseed fermented doughs used as inoculants were mixed with preactivated yeast and the remaining pre-mixed dry ingredients were finally added. The bread doughs were kneaded for $30 \mathrm{~s}$ at low speed and $90 \mathrm{~s}$ at medium speed in a Kitchen Aid mixer. The batter was then placed into $15 \mathrm{~cm} \times 8 \mathrm{~cm}$ fat-sprayed baking tins, covered by thermoresistant film and leave for fermentation at $30{ }^{\circ} \mathrm{C}$ for $90 \mathrm{~min}$ in a proofer (Cres-cor, Crescent Metal Products Inc, Ohio, USA). Breads were then baked in a deck oven at $350{ }^{\circ} \mathrm{C}$ for $35 \mathrm{~min}$. After baking, the loaves were depanned and cooled for $120 \mathrm{~min}$ at room temperature. Baking was performed on three different days (3 independent trials) and the obtained loaves were used for subsequent analyses.

\subsubsection{Bread specific volume}

The obtained breads were evaluated within $2 \mathrm{~h}$ after baking. Specific volume of bread loaves was determined by a modification of 10-05 AACC (2000) seed displacement method described by Lainez (2006). Instead of rapeseed, black pepper seeds were used in this study. The specific volume was calculated by dividing the volume by loaf weight and expressed as $\mathrm{cm}^{3} / \mathrm{g}$. This test was performed by triplicate.

\subsubsection{Sensory evaluation by consumer panel}

Sensory evaluation was assessed for adherence to ethical guidelines and approved by the Research Ethics Board at the University of Alberta (Pro00036093). The panel involving fifty-six non-trained panelists ( $54 \%$ male and $46 \%$ female) between 18 and 24 years old were recruited randomly from the Agricultural \& Forestry Centre of the University of Alberta and all participants expressed written informed consent. In order to get information about baked goods preference, an exploratory evaluation was first performed by asked panelists to answer three questions (how often is bread consumed; how much is bread liking and favorite baked good). In addition, an acceptance test was performed to rank sensory attributes among sorghum-based breads with 20, 30 and $40 \% W c$-chia sourdough replacements and $100 \%$ sorghum bread. The frozen breads were thawed overnight at room temperature before sensory evaluation. Chia inoculated breads were cut into slices (10 mm thickness) and slices (4) were placed separately in labeled plastic dishes and randomly assigned to each panelist. They were asked to rate sorghum breads with 0 (reference), 20, 30 and $40 \%$ chia sourdough replacement samples for taste, texture and global appearance by a 1-9 hedonic scale (9: like extremely, 8: like very much, 7: like moderately, 6: like slightly, 5: neither like nor dislike, 4: dislike slightly, 3: dislike moderately, 2: dislike very much, 1 : dislike extremely). Means from data were calculated \pm SEM. Moreover, the Duo-trio test as an affective discriminative test for overall differences was performed, to assess whether participants could differentiate between sorghum breads prepared using $20 \% W c$-inoculated chia sourdough or $20 \%$ unfermented chia dough. This replacement $\%$ was used because after $20 \%$ of replacement specific volume was near 2.0 (acceptable for bread). Three sorghum-based bread slices, involving a slice codded as R (bread inoculated with $20 \% W c$-chia sourdough) and two test slices (containing 20\% unfermented chia dough and 20\% Wc chia sourdough) were placed in plastic dishes. Participants were asked to identify which one of the two test samples is the reference. Sensory evaluation was performed within a single day in the sensory testing laboratory at the Department of Agricultural, Food and Nutritional Science, University of Alberta. The panelists were provided with a glass of water, pre-randomized bread slices, pencil and a questionnaire. Panelists were instructed to sip water for palate cleansing in between samples. Samples were presented to the evaluators blind so that the panels did not know which sample they were evaluating.

\subsection{Statistical analysis}

The software package MINITAB 14 (Minitab Inc., State College, PA, USA) using ANOVA General Linear Models followed by a Tukey's HSD test was applied, and $\mathrm{P}$ value of $<0.05$ was considered statistically significant. Unless otherwise stated, all values were the means of three independent trials \pm standard deviation. The result for paired comparison test was analyzed by T-test; for Just About Right scale result, the frequency of each category was calculated. For ranking result, the sum of the rank number for each sample (acceptable panelist answers for different sourdoughs replacement percentages divided by panelist's numbers) was calculated and the result was analyzed by the nonparametric Friedman method. For Duo-trio test, the number of correct responses was counted and used statistical chart 4 as described to determine if a detectable overall difference exists between sorghum-based breads with different chia-sourdough replacement percentage (Poste, Mackie, Butler, \& Larmond, 1991).

\section{Results and discussion}

\subsection{Chia and flaxseed sourdough fermentation}

Chia and flaxseed flours were used to prepare laboratory sourdoughs that served to inoculate gluten-free breads. After $24 \mathrm{~h}$ of fermentation at $30^{\circ} \mathrm{C}$, variable growth of $\mathrm{LAB}$ as determined by cell counts and dough's $\mathrm{pH}$ decrease, was observed. Cell counts increase and $\mathrm{pH}$ changes during fermentation was higher for chia compared to flaxseed sourdoughs (Table 2). However, all assayed strains and their combinations were able to acidify doughs; $\Delta \mathrm{pH}$ values ranged from 0.61 to 1.42 and 0.32 to 1.21 in chia and flaxseed sourdoughs, respectively; the greatest $\mathrm{pH}$ reduction was produced in doughs fermented with $L$. plantarum FUA3171 ( $L p$ ); heterofermentative W. cibaria CH28 (Wc) and L. fermentum FUA3165 ( $L f)$ also showed a $\Delta \mathrm{pH}$ greater than $1.0 \mathrm{log}$ $\mathrm{CFU} / \mathrm{g}$ at $24 \mathrm{~h}$. When compared to cereals and legumes flours fermentation (Rizzello, Calasso, Campanella, De Angelis, \& Gobbetti, 2014; Sáez et al., 2017), lower pH values and cell counts were showed in chia and flaxseed fermentation which is in agreement with the low carbohydrates concentration of oilseeds. However, the higher cell counts for $L p+L f, L f$ and $W c+L c l$ (Table 2), are in correlation with the ability of W. cibaria, L. fermentum and L. plantarum to use pentoses (xylose and arabinose) and hexoses (glucose, galactose and rhamnose) present in the doughs. Co-fermentation of hexoses and pentoses enhances cell yield and growth rate during fermentation, since more energy is produced by sourdough LAB during growth on pentoses than on hexoses; mixed obligate and facultative heterofermentative LAB ensured rapid growth and acidification (Coda, Rizzello, \& Gobbetti, 2010; Denkova et al., 2014b; Kim, Block, \& Mills, 2010). As reported for W. cibaria, it is able to display high acidification rate due to the ability to metabolize plant-derived polysaccharides such as arabinoxylans by $\beta$-xylosidase, xylanase, $\alpha$-arabinofuranosidase and feruloil oxidase activities, with high impact on biotechnological and nutritional features of foods by increasing soluble dietary fibers and free ferulic acid content (Katina et al., 2012; Lynch et al., 2015; Quattrini, Korcari, Ricci, \& Fortina, 2019). The presence of polysaccharides in chia and flaxseed seeds/ flours containing pentoses such as xylose and arabinose may have been 
Table 2

Growth characteristics and biochemical parameters of chia $(\mathrm{CH})$ and flaxseed (FL) sourdoughs after $24 \mathrm{~h}$ of fermentation at $30{ }^{\circ} \mathrm{C}$.

\begin{tabular}{|c|c|c|c|c|c|c|c|}
\hline \multirow[t]{2}{*}{ Parameters } & \multirow[t]{2}{*}{ Flour } & \multirow[t]{2}{*}{ Control $^{\mathrm{a}}$} & \multicolumn{5}{|l|}{ Inoculated LAB } \\
\hline & & & Wc & $\mathrm{Wc}+\mathrm{Lcl}$ & $\mathrm{Lp}$ & Lf & $\mathrm{Lp}+\mathrm{Lf}$ \\
\hline \multirow[t]{2}{*}{$\triangle \log \mathrm{CFU} / \mathrm{g}^{\mathrm{b}}$} & $\mathrm{CH}$ & $\mathrm{NR}^{\mathrm{d}}$ & $0.81 \pm 0.01$ & $1.15 \pm 0.02$ & $0.98 \pm 0.01$ & $1.43 \pm 0.03$ & $1.48 \pm 0.02$ \\
\hline & FLX & NR & $0.54 \pm 0.02$ & $0.98 \pm 0.01$ & $1.01 \pm 0.03$ & $1.08 \pm 0.02$ & $1.24 \pm 0.01$ \\
\hline \multirow[t]{2}{*}{$\triangle \mathrm{pH}^{\mathrm{c}}$} & $\mathrm{CH}$ & $0.11 \pm 0.01$ & $1.06 \pm 0.03$ & $0.61 \pm 0.02$ & $1.42 \pm 0.02$ & $1.01 \pm 0.01$ & $1.31 \pm 0.02$ \\
\hline & FLX & $0.11 \pm 0.01$ & $0.32 \pm 0.01$ & $0.41 \pm 0.01$ & $1.21 \pm 0.02$ & $0.62 \pm 0.02$ & $0.90 \pm 0.01$ \\
\hline \multicolumn{8}{|l|}{ Organic acids } \\
\hline \multirow[t]{2}{*}{ Lactic acid (mM) } & $\mathrm{CH}$ & $34.1 \pm 10.2$ & $25.1 \pm 1.3$ & $26.2 \pm 0.5$ & $22.4 \pm 1.5$ & $23.9 \pm 0.9$ & $\mathrm{ND}^{\mathrm{e}}$ \\
\hline & FLX & $27.5 \pm 9.5$ & $19.2 \pm 2.2$ & $21.3 \pm 1.5$ & $20.5 \pm 2.1$ & $19.7 \pm 1.5$ & ND \\
\hline \multirow[t]{2}{*}{ Acetic acid (mM) } & $\mathrm{CH}$ & $5.3 \pm 0.4$ & $4.5 \pm 0.5$ & $4.1 \pm 0.5$ & $4.0 \pm 0.2$ & $4.1 \pm 0.4$ & ND \\
\hline & FLX & $4.5 \pm 0.5$ & $3.4 \pm 0.4$ & $3.3 \pm 0.3$ & $3.1 \pm 0.4$ & $3.4 \pm 0.5$ & ND \\
\hline \multirow[t]{2}{*}{ Ethanol (mM) } & $\mathrm{CH}$ & 0.0 & $5.2 \pm 0.2$ & $4.5 \pm 0.5$ & $4.6 \pm 0.1$ & $6.6 \pm 0.5$ & ND \\
\hline & FLX & 0.0 & $3.3 \pm 0.4$ & $6.3 \pm 0.5$ & $7.2 \pm 0.4$ & $7.5 \pm 0.4$ & ND \\
\hline \multirow[t]{2}{*}{ FQ (lactate/acetate) } & $\mathrm{CH}$ & ND & 5.5 & 6.4 & 5.6 & 5.8 & ND \\
\hline & FLX & ND & 5.6 & 6.4 & 6.6 & 5.7 & ND \\
\hline \multicolumn{8}{|l|}{ Water soluble sugars } \\
\hline \multirow[t]{2}{*}{ Arabinose $(\mathrm{mM})$} & $\mathrm{CH}$ & $19.5 \pm 1.1$ & $17.7 \pm 1.1$ & $17.8 \pm 0.2$ & $11.4 \pm 0.6$ & $18.1 \pm 0.5$ & ND \\
\hline & FLX & $42.9 \pm 4.5$ & $82.1 \pm 2.1$ & $101.1 \pm 3.3$ & $71.3 \pm 4.3$ & $57.1 \pm 3.2$ & ND \\
\hline \multirow[t]{2}{*}{ Xylose (mM) } & $\mathrm{CH}$ & $57.4 \pm 0.7$ & $31.1 \pm 0.2$ & $29.4 \pm 0.2$ & $25.4 \pm 1.1$ & $32.9 \pm 2.1$ & ND \\
\hline & FLX & $84.2 \pm 4.2$ & $98.7 \pm 1.1$ & $112.8 \pm 6.4$ & $120.5 \pm 7.2$ & $81.1 \pm 3.2$ & ND \\
\hline \multirow[t]{2}{*}{ Rhamnose (mM) } & $\mathrm{CH}$ & 0.0 & 0.0 & 0.0 & 0.0 & 0.0 & ND \\
\hline & FLX & $2.8 \pm 0.1$ & $3.3 \pm 0.1$ & $3.5 \pm 0.2$ & $2.3 \pm 0.1$ & $3.2 \pm 0.1$ & ND \\
\hline \multirow[t]{2}{*}{ Free $\alpha$ - amino acids (mg FAA/100 g) } & $\mathrm{CH}$ & $10.91 \pm 0.01$ & $21.71 \pm 0.02$ & $36.42 \pm 0.02$ & $31.04 \pm 0.02$ & $34.88 \pm 0.02$ & $23.16 \pm 0.03$ \\
\hline & FLX & $12.51 \pm 0.02$ & $24.33 \pm 0.03$ & $31.25 \pm 0.01$ & $30.55 \pm 0.03$ & $30.78 \pm 0.03$ & $20.03 \pm 0.03$ \\
\hline \multirow{2}{*}{ Total peroxides $\left(\mathrm{mmol}_{2} \mathrm{O}_{2} / 100 \mathrm{~g}\right)$} & $\mathrm{CH}$ & $14.82 \pm 0.01$ & $26.31 \pm 0.01$ & $13.50 \pm 0.01$ & $19.28 \pm 0.01$ & $14.81 \pm 0.02$ & $16.38 \pm 0.02$ \\
\hline & FLX & $24.6 \pm 0.02$ & $28.51 \pm 0.01$ & $15.41 \pm 0.02$ & $18.7 \pm 0.02$ & $18.16 \pm 0.02$ & $23.89 \pm 0.03$ \\
\hline
\end{tabular}

All determinations were performed in triplicate. CH: chia; FLX: flax; Wc: Weisella cibaria CH28; Lcl: Lactococcus lactis CH179; Lp: Lactobacillus plantarum FUA3165; Lf: Lactobacillus fermentum FUA3171.

a Uninoculated and chemically acidified with lactic acid:acetic acid (4:1).

b $\mathrm{Log} \mathrm{CFU} / \mathrm{g}$ at $24 \mathrm{~h}-\log \mathrm{CFU} / \mathrm{g}$ at $0 \mathrm{~h}$.

c $\mathrm{pH}_{24}-\mathrm{pH}_{0}$.

d NR: not relevant.

e ND: not determined.

affected the sourdough fermentation.

Fermentation end-products present in sourdoughs inoculated by different LAB were assessed after $24 \mathrm{~h}$ (Table 2). As expected, lactate was the major metabolite in the doughs, its concentration ranged from $22.4 \pm 1.5$ to $25.1 \pm 1.3$ and $19.2 \pm 2.2$ to $21.3 \pm 1.5 \mathrm{mM}$ for chia and flaxseed sourdoughs, respectively while produced acetate was between $4.0 \pm 0.22$ and $4.5 \pm 0.5 \mathrm{mM}$ in chia sourdoughs and $3.1 \pm 0.5$ and $3.4 \pm 0.5 \mathrm{mM}$ in flaxseed sourdoughs. These results indicate that $W c$ inoculated in chia dough produced the maximum concentration of lactic and acetic acids. However, compared to Italian legumes fermentation (Curiel et al., 2015), lactic acid concentration after $24 \mathrm{~h}$ was substantially lower in both oilseeds while acetic acid showed to be higher. While acetic and lactic acid both contribute to acidity and texture of sourdough, the former is a flavor enhancer, antiropiness and antifungal compound (De Vuyst et al., 2014). Homofermentative $L c$. lactis virtually produce only lactic acid while heterofermentative $W$. cibaria and $L$. fermentum produce acetic acid, ethanol and $\mathrm{CO}_{2}$ in addition to lactic acid (von Wright \& Axelsson, 2019). However, facultative homofermentative $L$. plantarum that produce lactic acid from hexoses, can also ferment pentoses with an increased acetate production (Gänzle, Vermeulen, \& Vogel, 2007). Concerning ethanol production during oilseeds fermentation, higher values were obtained for flaxseed doughs. When fermentation quotient (FQ) values were calculated, a variation from 5.5 to 6.6 in oilseed dough's independently of the type of flour and inoculated LAB was found. FQ is highly dependent on the capacity to synthesize acetic acid, affecting the sensory characteristics of sourdoughs. FQ for sourdoughs should be in the range of 2.0-2.7 (Lim et al., 2018); in this study FQ values from heterofermentative $\mathrm{LAB}$ ( $W c$ and $L f$ ) reached values closer to optimum. Thus, fermented dough's with these bacteria would be more balanced regarding sensory, functional and safety characteristics.

On the other hand, water-soluble carbohydrates (WSC) released at
$24 \mathrm{~h}$ of fermentation in the doughs inoculated by different LAB were evaluated (Table 2). Chia seed water-soluble polysaccharide contains a repeating unit with a ratio of glucose:xylose:arabinose:galactose:mannose:glucuronic acid of 17:6:4:1:0.74:0.16, whereas that of flaxseed is composed by a neutral arabinoxylan (75\%) containing mainly xylose, arabinose and galactose and an acidic rhamnogalacturonan (25\%) consisting of rhamnose, galactose, galacturonic and fucose acid (Bernacchia et al., 2014; Goh et al., 2016). Polysaccharides solubilization in chia and flaxseed sourdoughs may be attributed to endogenous seed enzymes, mechanical disaggregation and acid hydrolysis as was previously reported for rye polysaccharide (Loponen et al., 2009). Among the analyzed carbohydrates, glucose was undetectable in WSC from fermented doughs indicating its consumption by inoculated LAB, however, concentrations of $13.7 \pm 1.5$ and $9.5 \pm 1.0 \mathrm{mM}$ for chia and flaxseed chemically acidified controls were determined. When the presence of arabinose, xylose and rhamnose, as main constituents of water soluble arabinoxylan and rhamnogalacturonan polysaccharides, was determined in chia and flaxseed fermented dough's, their concentrations were dependent of the dough matrix and inoculated LAB. Chia dough's exhibited lower arabinose $(11.4 \pm 0.6-18.1 \pm \pm \quad 0.5 \mathrm{mM})$ and xylose $(25.4 \pm 1.1-32.9 \pm 2.1 \mathrm{mM})$ contents when compared to chemically acidified control. On the other hand, a greater polysaccharides hydrolysis was observed in flaxseed sourdoughs after fermentation, concentrations of arabinose $(57.1 \pm 3.2-101.1 \pm 3.3 \mathrm{mM})$ and xylose $(81.1 \pm 3.2-120.5 \pm 7.2 \mathrm{mM})$ were even higher than that of chemically acidified control dough, this suggesting a greater polysaccharides solubilization (Table 2). In addition, a minor concentration of rhamnose was also detected in flaxseed dough's. These results must be considered as a balance between polysaccharides degradation by acid hydrolysis, bacterial enzymatic activity and sugars released by LAB to grow during dough's fermentation. Arabinoxylan as a major 


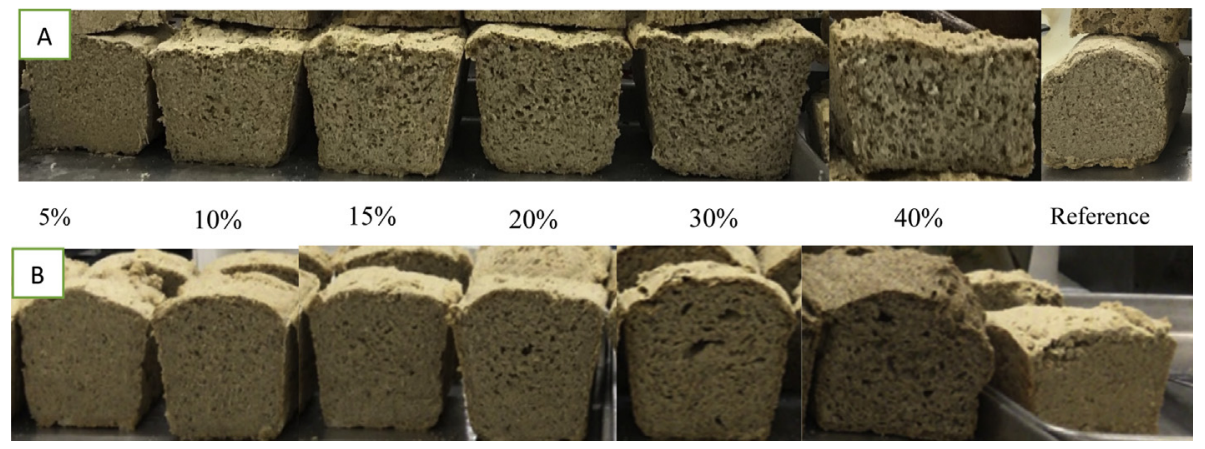

Fig. 1. Sorghum-based breads started with different percentages of chia and flaxseed sourdoughs inoculated with $W c(W$. cibaria $\mathrm{CH} 28)$ and $L p+L p$ (lactobacilli combination).

carbohydrate component of chia and flaxseed flours was reported to be degraded mostly by heterofermentative LAB (Michlmayr et al., 2013; Pontonio et al., 2016; Quattrini et al., 2019). Accordingly, all inoculated doughs were able to hydrolyze water-soluble polysaccharides during fermentation and part of the released sugars would have been metabolized by bacteria to growth; $W$. cibaria and Lc. lactis (from plant origin) were reported to metabolize arabinose and xylose (Björkroth \& Holzapfel, 2006; Golomb \& Marco, 2015). In addition, L. fermentum and L. plantarum are able to use arabinose but not xylose (Gobbetti, Lavermicocca, Minervini, De Angelis, \& Corsetti, 2000); this fact may explain the lower arabinose concentration compared to xylose that was accumulated in sourdoughs.

When protein degradation after $24 \mathrm{~h}$ of chia and flaxseed fermentation was evaluated (Table 2), higher content of free $\alpha$-amino acids (FAA) compared to the chemically acidified control were determined. FAA markedly increased in both oilseed dough's, greater amino acids accumulation being produced when $W c+L c l$ was inoculated $(36.42 \pm 0.02$ and $31.25 \pm 0.01 \mathrm{mg} \mathrm{FAA} / 100 \mathrm{~g}$ for chia and flaxseed dough's, respectively). However, Golomb and Marco (2015) reported the presence of genes encoding LPXTG motif-containing proteins in plant-origin $L c$. lactis strains with a possible role in vegetal proteins degradation. Amino acid concentration increased during fermentation is mainly due to the activity of strain-specific intracellular peptidases (Gänzle, 2014). Although cell envelope proteinase absence, W. cibaria was described to possess an extensive proteolytic system; fifteen putative peptidases were recognized and the three known LAB peptide transport systems were identified reflecting its auxotrophy and adaptation to peptide-rich sourdough environment (Lynch et al., 2015). Similar to that found for sugars concentration in sourdoughs, FAA concentration at $24 \mathrm{~h}$ also indicate a balance between proteins/peptides degradation and consumption by bacteria for growth. Peptides and amino acids from plant and bacterial enzymatic activity would have an impact on bread quality as taste-active compounds, flavor precursors or as bioactives.

Furthermore, during kneading, lipids oxidation generates volatile flavor compounds and influences the rheology of the dough (Gänzle, 2014). During mixing of wheat and rye doughs, oxygen is consumed by seeds endogenous lipoxygenase activity which oxidize linoleic acid to hydroxyperoxy acids and depending on oxygen availability they will further be degraded by enzymatic and non-enzymatic to aldehydes with high influence on flavor (Belitz, Grosch, \& Schieberle, 2004, pp. 218-219). When peroxide concentration in sourdoughs was determined after $24 \mathrm{~h}$ of fermentation (Table 2), the highest release was produced in both sourdoughs inoculated with $W c(26.31 \pm 0.01$ and $28.51 \pm 0.01 \mathrm{mmol} / 100 \mathrm{~g}$ dough, respectively) compared to chemically acidified dough's; their accumulation increasing by $44 \%$ and $14 \%$ in chia and flaxseed, respectively. The presence of $\mathrm{H}_{2} \mathrm{O}_{2}$ in doughs after fermentation is related to oxygen effect on the fate of pyruvate in LAB. Oxygen participate in $\mathrm{LAB}$ metabolism as external electron acceptor through NADH oxidase forming $\mathrm{H}_{2} \mathrm{O}_{2}$, this being demonstrated for heterofermentative LAB which increase their growth under aerated conditions with the production of acetate instead of ethanol (von Wright \& Axelsson, 2019). LAB-generated $\mathrm{H}_{2} \mathrm{O}_{2}$ in the dough's is thought to exert an antimicrobial effect with the additional contribution to a stronger and more elastic structure and water absorption (Nami, Gharekhani, Aalami, \& Hejazi, 2019).

\subsection{Gluten-free breads}

Based on the cell density, highest acidification and lactic/acetic acids production, $W c$ and $L p+L f$ fermented chia and flaxseed sourdoughs were selected as inoculants for GF breads formulation (Table 1); $L p+L f$ as inoculants being used for comparative purposes. As reported by Sandri et al. (2017), physical properties and visual appearance showed whole chia flour alone as unsuitable for sourdough and bread production, however partial addition of oleaginous seed/flour to gluten-free flours would allow obtaining baked products with improved nutritional traits and acceptable sensory characteristics. Thus, experimental GF breads formulated with sorghum flour and including different proportions (5-40\%) of chia and flaxseed sourdoughs fermented by $W c$ and lactobacilli $(L p+L f)$ as ingredients, were produced and compared to breads inoculated with the same percentages of non-fermented oilseed doughs as well as sorghum (100\%) bread. After baking, obtained loaves (Fig. 1) were analyzed for their visual appearance and specific volume (SV). This physical trait has been adopted in the literature as the most reliable measure for bread. Higher weights and volumes exert positive economic effect for breads production because consumers are often attracted by bread loaves with higher volume; thus, reduction of loaf size during the baking process is undesirable (Costantini et al., 2014). SV of sorghum-based breads started with different percentages of $W c$ - and lactobacilli-inoculated chia and flaxseed sourdoughs and unfermented flours are shown in Table 3. Results indicated non-significant differences between fermented and unfermented sorghum-based loaves started with $5-15 \%$ of oilseeds dough's (data not shown) and when comparisons along the rows were

Table 3

Specific volume $\left(\mathrm{cm}^{3} / \mathrm{g}\right)$ of sorghum-based breads started with $W$. cibaria and lactobacilli $(L p+L f)$ sourdoughs.

\begin{tabular}{|c|c|c|c|c|}
\hline Sourdough (\%) & Flour & $W c$ & $L p+L f$ & Unfermented flours \\
\hline \multirow[t]{2}{*}{0 (control) } & $\mathrm{CH}$ & $1.61 \pm 0.11^{\mathrm{a}}$ & $1.61 \pm 0.11^{\mathrm{a}}$ & $1.61 \pm 0.11^{\mathrm{a}}$ \\
\hline & FL & $1.61 \pm 0.11^{\mathrm{a}}$ & $1.61 \pm 0.11^{\mathrm{a}}$ & $1.61 \pm 0.11^{\mathrm{a}}$ \\
\hline \multirow[t]{2}{*}{20} & $\mathrm{CH}$ & $1.98 \pm 0.10^{\mathrm{b}}$ & $1.88 \pm 0.05^{\mathrm{ab}}$ & $1.85 \pm 0.06^{\mathrm{b}}$ \\
\hline & FLX & $1.85 \pm 0.42^{\mathrm{b}}$ & $1.79 \pm 0.17^{\mathrm{ab}}$ & $1.87 \pm 0.11^{\mathrm{ab}}$ \\
\hline \multirow[t]{2}{*}{30} & $\mathrm{CH}$ & $2.08 \pm 0.10^{\mathrm{b}}$ & $1.92 \pm 0.05^{\mathrm{b}}$ & $1.89 \pm 0.27^{\mathrm{bc}}$ \\
\hline & FLX & $1.98 \pm 0.09^{\mathrm{b}}$ & $1.95 \pm 0.11^{\mathrm{b}}$ & $1.85 \pm 0.12^{\mathrm{ab}}$ \\
\hline \multirow[t]{2}{*}{40} & $\mathrm{CH}$ & $2.15 \pm 0.15^{\mathrm{c}}$ & $2.12 \pm 0.07^{\mathrm{b}}$ & $1.84 \pm 0.13^{\mathrm{b}}$ \\
\hline & FLX & $2.01 \pm 0.08^{\mathrm{b}}$ & $2.08 \pm 0.26^{\mathrm{c}}$ & $1.80 \pm 0.13^{\mathrm{b}}$ \\
\hline
\end{tabular}

Specific volume was expressed as means $\pm \mathrm{SD}$; a, b, c within columns indicate a significant difference $(P \leq 0.05)$ between values based on a one-way ANOVA. 
carried out. However, SV of breads were significantly increased ( $>2.00 \mathrm{~cm}^{3} / \mathrm{g}$ ) when a replacement of 30 and $40 \%$ of $W c$-chia sourdoughs was performed. Maximal specific volumes were achieved when $40 \%$ of sorghum flour was replaced with $W c$ and lactobacilli inoculated chia sourdoughs $\left(2.15 \pm 0.15\right.$ and $2.12 \pm 0.07 \mathrm{~cm}^{3} / \mathrm{g}$, respectively). A correlation between the increase of oilseeds sourdough proportion in sorghum-based loaves and SV volume, was found. Similarly, 10-14\% chia flour addition allowed for a slight increase in the SV of wheat and rice breads (Costantini et al., 2014; Sandri et al., 2017). Contrarily, a decrease of loaf SV of breads containing legumes mix or chia flours was found at percentages higher than $15 \%$ when added to a regular wheat and rice bread formulation (Rizzello et al., 2014; Steffolani, de la Hera, Pérez, \& Gómez, 2014). When compared SV found in this study with that of traditional wheat bread fermented by yeast $>3 \mathrm{~cm}^{3} / \mathrm{g}$ as reported by Rizzello et al. (2014), values $<2.5 \mathrm{~cm}^{3} / \mathrm{g}$ exhibited by sorghum-based breads would be related with a lower porosity and higher crumb firmness. However, the beneficial effect of oilseeds rich in arabinoxylans on dough's rheology affecting products volume compensate somewhat gluten absence during fermentation, slowing down $\mathrm{CO}_{2}$ diffusion rate out of the dough, thus contributing to gas retention (Lamo \& Gómez, 2018). Moreover, visual appearance of sorghum-based breads inoculated by chia and flaxseed fermented sourdough exhibited an intense color of the bread crumb and crust, that of breads inoculated with chia sourdough were darker than those inoculated by flaxseed sourdough. Dark color increase is in correlation with the replacement percentage, $40 \%$ of chia sourdough showing the darkest color (Fig. 1). This darkening effect was also reported in other studies for baked products involving chia seeds/flour addition (Costantini et al., 2014; Sandri et al., 2017; Steffolani et al., 2014). The color of the bread crumb mainly depends on raw materials used, in this study both sorghum and chia seed/flour are rich in phenolic compounds which besides their antioxidant properties affect the color of the bread (Iglesias-Puig \& Haros, 2013).

\subsection{Sensory evaluation}

To complement physical acceptability of sorghum-based breads, sensory evaluation was carried out. First, an exploratory assessment to investigate consumer preferences for bakery goods was conducted. Questionnaire results showed that $47.27 \%$ of the participants liked bread, $44.64 \%$ answered to consume bread 2-3 times a week, while breads and cakes $(26.79 \%$ each) were chosen as favorites among baked goods (Table 4). Then, a sensory evaluation with a consumer panel (56) who usually consume bread, was performed to assess the effect of sorghum flour replacement level by 20,30 and $40 \%$ of $W c$-fermented chia sourdoughs and to indicate the most accepted. The sensory attributes taste, texture and overall appearance were evaluated with obtained sorghum-based breads or control bread produced without sourdough (Table 5). Participants scored sensory attributes with a maximal value of five (neither like nor dislike) for breads produced with $40 \%$ of chia sourdough; those with $30 \%$ of replacement being less preferred (dislike slightly). Loaves with $100 \%$ of sorghum flour (reference) showed texture and overall aspect scored with 2.9 and 3.2 (dislike

Table 4

Baked goods preference.

\begin{tabular}{llllll}
\hline $\begin{array}{l}\text { How often do you } \\
\text { consume bread? }\end{array}$ & $\%$ & $\begin{array}{l}\text { How much do } \\
\text { you like bread? }\end{array}$ & $\%$ & $\begin{array}{l}\text { Favorite bakery } \\
\text { products }\end{array}$ & $\%$ \\
\hline $\begin{array}{l}\text { Once a day } \\
\text { 2-3 times per week }\end{array}$ & 32.14 & Like very much & 29.09 & Bread & 26.79 \\
Once a week & 8.93 & Like & 47.27 & Cakes & 26.79 \\
& & dislike & & Cookies & 14.29 \\
Every 2-3 weeks & 14.29 & Dislike & 1.82 & Donuts & 17.86 \\
Never & - & & & $\begin{array}{l}\text { Other (pretzels, } \\
\text { muffins, bagels) }\end{array}$ & 14.27 \\
& & & & 21.82 & \\
\hline
\end{tabular}

Table 5

Sensory evaluation of sorghum breads with W. cibaria CH28-chia sourdoughs.

\begin{tabular}{llll}
\hline $\begin{array}{l}\text { Chia sourdough } \\
\%(\mathrm{w} / \mathrm{w})\end{array}$ & Taste & Texture & Overall aspect \\
\hline 0 (Reference) & $3.8 \pm 0.23$ & $2.9 \pm 0.21$ & $3.2 \pm 0.22$ \\
20 & $4.0 \pm 0.23$ & $3.9 \pm 0.23$ & $3.8 \pm 0.22$ \\
30 & $4.8 \pm 0.23$ & $4.8 \pm 0.23$ & $4.5 \pm 0.23$ \\
40 & $5.0 \pm 0.22$ & $5.0 \pm 0.24$ & $5.0 \pm 0.23$ \\
\hline
\end{tabular}

moderately) and taste with 3.8 (dislike slightly). SEM values for all samples are very similar, ranging only from 0.21 to 0.24 . Consumer panelists perceived a bread firmness reduction showing a "soggy" texture, although despite these complaints, bread acceptability increased as chia sourdough proportion increased, $40 \%$ of replacement being the most preferred. In view to detect differences in treatments with $\mathrm{Wc}$ fermented chia sourdoughs across test attempts, the non-parametric Friedman test allowed breads to be separate in two groups. Statistically significant difference $(\mathrm{P}<0.05)$ was established between them, sorghum-based breads produced with 30 and $40 \%$ chia sourdoughs being the most accepted by panelists (52 and $26 \%$, respectively) while $11 \%$ panelists each preferred breads with 0 and $20 \%$ of sorghum flour replacement (Fig. 2). Moreover, Duo-trio test for overall difference discrimination was able to differentiate among breads inoculated with $\mathrm{Wc}$ fermented chia sourdough and unfermented chia. Thus, $70 \%$ of panelists (39 out of 56) were able to discriminate between inoculated bread (20\%) and unfermented (P: 0.008), these results indicating they were unlikely to be due to random chance (data not shown). Similar to this study, the use of legumes mix and quinoa sourdoughs fermented with autochthonous LAB to inoculate wheat flour bread were reported to have good acceptability due to sourdough fermentation, leading to an increase in the scores of several attributes compared to wheat bread (Rizzello et al., 2014; Rizzello et al., 2016). Fermentation has been already reported as a potential tool to improve technological and sensory characteristics of food. Sourdough fermentation is one of the oldest food biotechnologies, which was recently rediscovered for its positive effect on sensory, structure, nutritional and shelf-life features of leavened baked goods; the advantages attributed to sourdough is mostly ascribed to LAB selected for different biochemical and technological traits that allows to get a successful sourdough fermentation (Gobbetti, Rizzello, Di Cagno, \& De Angelis, 2014; Ruiz Rodríguez et al., 2016). The incorporation of chia sourdough to GF cereals-based breads has a number of advantages representing a step forward in the production of high quality, natural and healthy products.

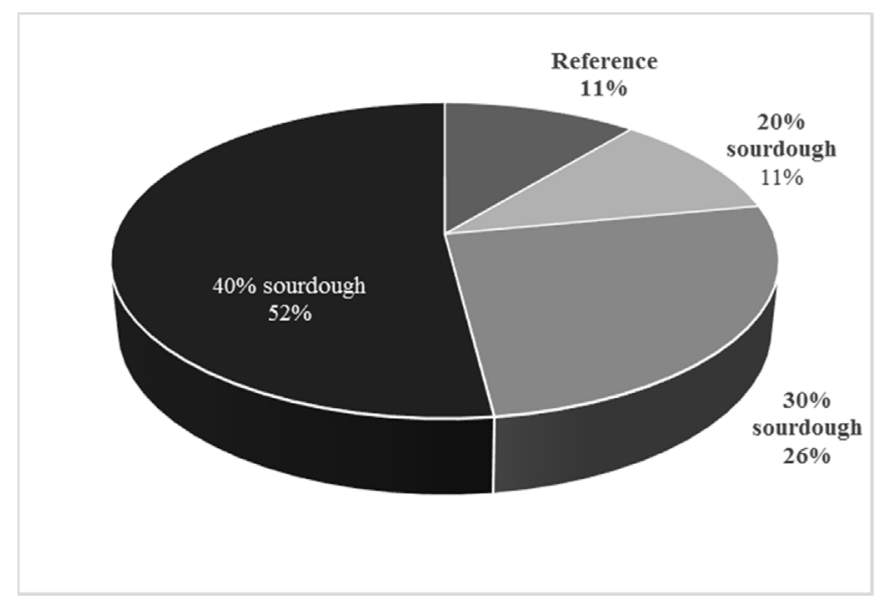

Fig. 2. Panelist's preference of sorghum-based breads distributed according to percentages of chia sourdough inoculated with $W$. cibaria $\mathrm{CH} 28(0 \%, 20 \%, 30 \%$ and $40 \%)$. 


\section{Conclusion}

Sorghum-based breads inoculated with chia and flaxseed sourdoughs used as a replacement for commonly used ingredients such as refined flours and starches, were obtained. Technological quality of loaves was affected by adding selected autochthonous LAB fermented oilseed sourdoughs, this leading to a significant increase in the specific volume of sorghum-based breads started with $30 \%$ and $40 \%$ chia and flaxseed sourdoughs. Sorghum-based GF breads inoculated with chia sourdoughs fermented with $W$. cibaria $\mathrm{CH} 28$ in a ratio of $40 \%$, showed the highest sensory features. The use of chia sourdough as ingredient for gluten-free breadmaking will contribute to increase proteins, dietary fiber and essential fatty acids, such as $\omega-3$ linolenic acid content while reducing the intake of saturated fatty acids.

\section{CRediT authorship contribution statement}

Stefanía Dentice Maidana: Investigation. Susan Finch: Methodology. Marisa Garro: Methodology. Graciela Savoy: Conceptualization, Writing - review \& editing. Michael Gänzle: Conceptualization, Funding acquisition, Supervision. Graciela Vignolo: Conceptualization, Funding acquisition, Writing - original draft, Writing - review \& editing.

\section{Declaration of competing interest}

None.

\section{Acknowledgements}

This work was supported by a grant from Consejo Nacional de Invetigaciones Científicas y Técnicas (CONICET), Argentina (PIP 2015 441). Michael Gänzle acknowledge the Natural Science and Engineering Research Council and the Canada Research Chairs for funding.

\section{References}

AACC. American Association of Cereal Chemist. (2000). Approved methods of the AACC $\left(10^{\text {th }}\right.$ ed.). St. Paul, MN. USA: The American Association of Cereal Chemists.

Ayerza, R., \& Coates, W. (2005). Ground chia seed and chia oil effects on plasma lipids and fatty acids in the rat. Nutrition Research, 25, 995-1003. https://doi.org/10.1016/ j.nutres.2005.09.013.

Belitz, H. D., Grosch, W., \& Schieberle, P. (2004). Food chemistry (3rd ed.). Berlin: Springer-Verlag.

Bernacchia, R., Preti, R., \& Vinci, G. (2014). Chemical composition and health benefits of flaxseed. Austin Journal of Nutrition and Food Sciences, 2(8), 1045.

Björkroth, J., \& Holzapfel, W. (2006). Genera leuconostoc, oenococcus and Weissella. Prokariotes, 4, 267-319. https://doi10.1007/0-387-30744-3_9.

Coda, R., Cagno, R. D., Gobbetti, M., \& Rizzello, C. G. (2014). Sourdough lactic acid bacteria: Exploration of non-wheat cereal-based fermentation. Food Microbiology, 37, 51-58. https://doi.org/10.1016/j.fm.2013.06.018.

Coda, R., Rizzello, C. G., \& Gobbetti, M. (2010). Use of sourdough fermentation and pseudo-cereals and leguminous flours for the making of a functional bread enriched of $\gamma$-aminobutyric acid (GABA). International Journal of Food Microbiology, 137, 236-245. https://doi10.1016/j.ijfoodmicro.2009.12.010.

Costantini, L., Lukšič, L., Molinari, R., Kreft, I., Bonafaccia, G., Manzi, L., et al. (2014). Development of gluten-free bread using tartary buckwheat and chia flour rich in flavonoids and omega-3 fatty acids as ingredients. Food Chemistry, 165, 232-240. https://doi.org/10.1016/j.foodchem.2014.05.095.

Curiel, J. A., Coda, R., Centomani, I., Summo, C., Gobbetti, M., \& Rizzello, C. G. (2015). Exploitation of the nutritional and functional characteristics of traditional Italian legumes: The potential of sourdough fermentation. International Journal of Food Microbiology, 196, 51-61. https://doi.org/10.1016/j.ijfoodmicro.2014.11.032.

De Vuyst, L., Van Kerrebroeck, S., Harth, H., Huys, G., Daniel, H. M., \& Weckx, S. (2014). Microbial ecology of sourdough fermentations: Diverse or uniform? Food Microbiology, 37, 11-29. https://doi.org/10.1016/j.fm.2013.06.002.

Denkova, R., Ilieva, S., Denkova, Z., Georgieva, L., \& Krastanov, A. (2014a). Examination of the technological properties of newly isolated strains of the genus Lactobacillus and possibilities for their application in the composition of starters. Biotechnology \& Biotechnological Equipment, 28(3), 487-494. https://doi.org/10.1080/13102818. 2014.918701.

Denkova, R., Ilieva, S., Denkova, Z., Georgieva, L., Yordanova, M., Nikolova, D., et al. (2014b). Production of wheat bread without preservatives using sourdough starters.
Biotechnology \& Biotechnological Equipment, 28(5), 889-898. https://doi.org/10. 1080/13102818.2014.965057.

Dentice Maidana, S., Aristimuño Ficoseco, C., Bassi, D., Puglisi, E., Savoy, G., Vignolo, G., et al. (2020). Chia as a new ingredient of sourdough fermentation and source of microbial diversity. International Journal of Food Microbiology, 316, 108425. https:// doi.org/10.1016/j.ijfoodmicro.2019.108425.

Gänzle, M. G. (2014). Enzymatic and bacterial conversions during sourdough fermentation. Food Microbiology, 37, 2-10. https://doi.org/10.1016/j.fm.2013.04.007.

Gänzle, M., Vermeulen, N., \& Vogel, R. F. (2007). Carbohydrate, peptide and lipid metabolism of lactic acid bacteria in sourdough. Food Microbiology, 24, 128-138. https://doi10.1016/j.fm.2006.07.006.

Gänzle, M. G., \& Zheng, J. (2018). Lifestyles of sourdough lactobacilli - do they matter for microbial ecology and bread quality? International Journal of Food Microbiology, S0168-1605(18), 30542-30547. https://doi.org/10.1016/j.ijfoodmicro.2018.08. 019.

Gobbetti, M., Lavermicocca, P., Minervini, F., De Angelis, M., \& Corsetti, A. (2000). Arabinose fermentation by Lactobacillus plantarum in sourdough with added pentosans and alpha, alpha-L-arabinofuranosidase: A tool to increase the production of acetic acid. Journal of Applied Microbiology, 88, 317-324. https://doi.org/10.1046/j. 1365-2672.2000.00962x.

Goh, K. K. T., Matia-Merino, L., Chiang, J. H., Quek, R., Soh, S. J. B., \& Lentle, R. G. (2016). The physicochemical properties of chia seed polysaccharide and its microgel dispersion rheology. Carbohydrate Polymers, 149, 297-307. https://doi.org/10.1016/ j.carbpol.2016.04.126.

Golomb, B. L., \& Marco, M. L. (2015). Lactococcus lactis metabolism and gene expression during growth on plant tissues. Journal of Bacteriology, 197(2), 371-381. https://doi. org/10.1128/jb.02193-14.

Goyal, A., Sharma, V., Upadhyay, N., Gill, S., \& Sihag, M. (2014). Flax and flaxseed oil: An ancient medicine \& modern functional food. Journal of Food Science \& Technology, 51(9), 1633-1653. https://doi.org/10.1007/s13197-013-1247-9.

Hussain, S., Anjum, F. M., Alamri, M. S., Mohamed, A. A., \& Nadeem, M. (2013). Functional flaxseed in baking. Quality Assurance and Safety of Crops \& Foods, 5(4), 375-385. https://doi.org/10.3920/QAS2012.0207.

Iglesias-Puig, E., \& Haros, C. M. (2013). Evaluation of performance of dough and bread incorporating chia (Salvia hispanica L.). European Food Research and Technology, 237(6), 865-874. https://doi.org/10.1007/s00217-013-2067-x.

Katina, K., Juvonen, R., Laitila, A., Flander, L., Nordlund, E., Kariluoto, S., et al. (2012). Fermented wheat bran as a functional ingredient in baking. Cereal Chemistry, 89, 126-134. https://doi.org/10.1094/CCHEM-08-11-0106.

Kim, J.-H., Block, D. E., \& Mills, D. A. (2010). Simultaneous consumption of pentose and hexose sugars: An optimal microbial phenotype for efficient fermentation of lignocellulosic biomass. Applied Microbiology and Biotechnology, 88, 1077-1085. https:// doi10.1007/s00253-010-2839-1.

Lainez, E. (2006). Estudio de la estabilidad del pan parcialmente horneado conservado en refrigeración. Puebla. México: Tesis de Licenciatura. Universidad de las Américas.

Lamo, B., \& Gómez, M. (2018). Bread enrichment with oilseeds. A Review. Foods, 7, 191. https://doi.org/10.3390/foods7110191.

Lim, S. B., Tingirikari, J. M. R., Seo, J. S., Li, L., Shim, S., Seo, J.-H., et al. (2018). Isolation of lactic acid bacteria starters from Jeung-pyun for sourdough fermentation. Food Science and Biotechnology, 27(1), 73-78. https://doi.org/10.1007/s10068-017 0274-0.

Loponen, J., Kanerva, P., Zhang, C., Sontag-Strohm, T., Salovaara, H., \& Gänzle, M. G. (2009). Prolamin hydrolysis and pentosan solubilisation in germinated-rye sourdoughs determined by chromatographic and immunological methods. Journal of Agricultural and Food Chemistry, 57, 746-753. https://doi.org/10.1021/jf803243w.

Lynch, K. M., Lucid, A., Arendt, E. K., Sleator, R. D., Luce, B., \& Coffey, A. (2015). Genomics of Weissella cibaria with an examination of its metabolic traits. Microbiology, 161, 914-930. https://doi.org/10.1099/mic.0.000053.

Marineli, R. S., Moraes, É. A., Lenquiste, S. A., Godoy, A. T., Eberlin, M. N., \& Maróstica, M. R., Jr. (2014). Chemical characterization and antioxidant potential of Chilean chia seeds and oil (Salvia hispanica L.). Lebensmittel-Wissenschaft und -Technologie- Food Science and Technology, 59(2), 1304-1310. https://doi.org/10.1016/j.lwt.2014.04. 014.

Meroth, C. B., Walter, J., Hertel, C., Brandt, M. J., \& Hammes, W. P. (2003). Monitoring the bacterial population dynamics in sourdough fermentation processes by using PCR- denaturing gradient gel electrophoresis. Applied and Environmental Microbiology, 69(1), 475-482. https://doi.org/10.1128/AEM.69.1.475-482.2003.

Michlmayr, H., Hell, J., Lorenz, C., Böhmdorfer, S., Rosenau, T., \& Kneifel, W. (2013) Arabinoxylan oligosaccharide hydrolysis by family 43 and 51 glycosidases from Lactobacillus brevis DSM 20054. Applied and Environmental Microbiology, 79(21), 6747-6754. https://doi.org/10.1128/aem.02130-13.

Mohammad, A. (2015). Chemistry and antioxidant activity of plants containing some phenolic compounds. Chemistry International, 1(1), 35-52.

Moroni, A. V., Dal Bello, F., \& Arendt, E. K. (2009). Sourdough in gluten-free breadmaking: An ancient technology to solve a novel issue? Food Microbiology, 26, 676-684. https://doi.org/10.1016/j.fm.2009.07.001.

Muñoz, L. A., Cobos, A., Diaz, O., \& Aguilera, J. M. (2013). Chia seed (Salvia hispanica): An ancient grain and a new functional food. Food Reviews International, 29, 394-408. https://doi.org/10.1080/87559129.2013.818014.

Nami, Y., Gharekhani, M., Aalami, M., \& Hejazi, M. A. (2019). Lactobacillus-fermented sourdoughs improve the quality of gluten-free bread made from pearl millet flour. Journal of Food Science \& Technology, 56(9), 4057-4067. https://doi.org/10.1080/ 87559129.2013 .818014$.

Parker, J., Schellenberger, A. N., Roe, A. L., Oketch-Rabah, H., \& Calderón, A. I. (2018), Therapeutic perspectives on chia seed and its oil: A review. Planta Medica, 84, 606-612. https://doi.org/10.1055/a-0586-4711. 
Pontonio, E., Mahony, J., Di Cagno, R., O'Connell Motherway, M., Lugli, G. A., O'Callagha, A., et al. (2016). Cloning, expression and characterization of a $\beta$-d-xylosidase from Lactobacillus rossiae DSM $15814^{\mathrm{T}}$. Microbial Cell Factories, 15, 72 https://doi.org/10.1186/s12934-016-0473-z.

Poste, L. M., Mackie, D. A., Butler, G., \& Larmond, E. (1991). Laboratory methods for sensory analysis of food. Ottawa: Canada Department of Agriculture, Research Branch (Publication 1864/E 0-660- 13807-7.

Quattrini, M., Korcari, D., Ricci, G., \& Fortina, M. G. (2019). A polyphasic approach to characterize Weissella cibaria and Weissella confusa strains. Journal of Applied Microbiology. (in press) https://doi:10.1111/jam.14483.

Rizzello, C. G., Calasso, M., Campanella, D., De Angelis, M., \& Gobbetti, M. (2014). Use of sourdough fermentation and mixture of wheat, chickpea, lentil and bean flours for enhancing the nutritional, texture and sensory characteristics of white bread. International Journal of Food Microbiology, 180, 78-87. https://doi.org/10.1016/j. ijfoodmicro.2014.04.005.

Rizzello, C. G., Lorusso, A., Montemurro, M., \& Gobbetti, M. (2016). Use of sourdough made with quinoa (Chenopodium quinoa) flour and autochthonous selected lactic acid bacteria for enhancing the nutritional, textural and sensory features of white bread. Food Microbiology, 56, 1-13. https://doi.org/10.1016/j.fm.2015.11.018.

Ruiz Rodríguez, L., Vera Pingitore, E., Rollan, G., Cocconcelli, P. S., Fontana, C., Saavedra, L., et al. (2016). Biodiversity and technological-functional potential of lactic acid bacteria isolated from spontaneously fermented quinoa sourdoughs. Journal of Applied Microbiology, 120, 1289-1301. https://doi:10.1111/jam.13104.

Sáez, G. D., Hébert, E. M., Saavedra, L., \& Zárate, G. (2017). Molecular identification and technological characterization of lactic acid bacteria isolated from fermented kidney beans flours (Phaseolus vulgaris $\mathrm{L}$. and P. coccineus) in northwestern Argentina. Food Research International, 102, 605-615. https://doi.org/10.1016/j.foodres.2017.09. 042.
Sandri, L. T. B., Santos, F. G., Fratelli, C., \& Capriles, V. D. (2017). Development of glutenfree bread formulations containing whole chia flour with acceptable sensory properties. Food Sciences and Nutrition, 5(5), 1021-1028. https://doi.org/10.1002/fsn3. 495

Sekwati-Monang, B., \& Gänzle, M. G. (2011). Microbiological and chemical characterization of ting, a sorghum-based sourdough product from Botswana. International Journal of Food Microbiology, 150(2-3), 115-121. https://doi.org/10.1016/j. ijfoodmicro.2011.07.021.

Sinha, A. K. (1972). Colorimetric assay of catalase. Analytical Biochemistry, 47, 389-394. https://doi.org/10.1016/0003-2697(72)90132-7.

Steffolani, E., de la Hera, E., Pérez, G., \& Gómez, M. (2014). Effect of chia (Salvia Hispanica L) addition on the quality of gluten-free bread. Journal of Food Quality, 37, 309-317.

Thiele, C., Gänzle, M. G., \& Vogel, R. F. (2002). Contribution of sourdough lactobacilli, yeast, and cereal enzymes to the generation of amino acids in doughs relevant for bread flavor. Cereal Chemistry, 79(1), 45-51.

Wang, H., Wang, J., Qiu, C., Ye, Y., Guo, X., Chen, G., et al. (2017). Comparison of phytochemical profiles and health benefits in fiber and oil flaxseeds (Linum usitatissimum L.). Food Chemistry, 214, 227-233. https://doi.org/10.1016/j.foodchem. 2016.07.075.

von Wright, A., \& Axelsson, L. (2019). Lactic acid bacteria: An introduction. In G. Vinderola, A. C. Ouwehand, S. Salminen, \& A. von Wright (Eds.). Lactic acid bacteria Microbiological and functional aspects (pp. 1-16). Broken Sound Parway, NW: Taylor \& Francis Group.

Zannini, E., Jones, J. M., Renzetti, S., \& Arendt, E. K. (2012). Functional replacements for gluten. Annual Review in Food Science and Technology, 3, 227-245. https://doi.org/10 1146/annurev-food-022811-101203. 